\title{
Insight from Patients and Healthcare professionals on the implementation of virtual clinics in inflammatory bowel disease patients.
}

Aditi Kumar ${ }^{1 *}$, Mohammed Nabil Quraishi ${ }^{2}$, Shanika de Silva ${ }^{3}$, Nigel Trudgill ${ }^{4}$, Helen Steed ${ }^{1,5}$, Matthew J Brookes ${ }^{1,6^{* *}}$, Rachel M Cooney ${ }^{2 * *}$

1- The Royal Wolverhampton NHS Trust, Wolverhampton UK

2- University Hospital Birmingham NHS Trust Foundation, Birmingham UK

3- Dudley Group NHS Foundation Trust, Dudley UK

4- Sandwell and West Birmingham NHS Trust, Birmingham UK

5- Faculty of Sciences and Engineering, University of Wolverhampton, Wolverhampton UK

6- Research Institute in Healthcare Science (RIHS), University of Wolverhampton, Wolverhampton UK

*Corresponding author: aditikumar@nhs.net

*Joint lead authors

Abstract:

Introduction: During COVID-19, the management of outpatient inflammatory bowel disease (IBD) patients changed from face to face (F2F) to telephone and video consultations across the UK. We surveyed IBD patients and IBD Healthcare professionals (HCPs) to evaluate the impact of this abrupt transition on patient and HCP satisfaction outcomes, including the barriers and enablers of this service.

Methods: Patient satisfaction surveys were sent to patients who had a telephone consultation from May to July 2020. A second survey was sent to IBD HCPs across the UK. Questions from both surveys consisted of a mixture of multiple-choice options, ranking answers as well as short answer questions.

Results: 210 patients and 114 HCPs completed the survey. During COVID, there was a significantly greater use of telephone, video or a mixture of consultation. F2F consultations were consistently preferred by patients, with $50 \%$ of patients indicating they did not want the option of for video consultations. Patients were more likely to prefer a telephone consultation 
if they were stable and needed routine review. Significantly fewer HCPs (5.3\%) intend to use F2F consultations alone, preferring the use of telephone $(20.2 \%)$ or combinations of telephone/F2F (22.8\%), telephone/video $(4.4 \%)$ or combination of all three consultation types (34.2\%). 63\% indicated they intend to incorporate video consultations in the future.

Conclusion: Telephone and video consultations need to be balanced proportionately with F2F clinics to achieve both patient and HCP satisfaction. Further research needs to be done to explore the use of video medicine in IBD patients. 
Summary box:

What is already known about this subject:

1) Inflammatory bowel disease (IBD) patients are traditionally monitored three to sixmonthly in face to face (F2F) outpatient clinics regardless of disease activity, which use a significant proportion of outpatient capacity

2) There is increasing pressures within the healthcare system to reduce the increasing pressures on outpatient resources whilst maintaining a high quality of care towards patients which is safe and cost-effective

3) Implementation of virtual clinics encompassing telephone and/or video clinics has previously shown to reduce this burden

What are the new findings

1) IBD patients prefer F2F consultations over other modes of consultations, although would continue to use telephone services if offered

2) Video consultations were the least preferred modality for IBD patients

3) IBD Health care professionals (HCPs) intend to combine a mixture of F2F, video and telephone consultations to maintain future outpatient services, with over $63 \%$ indicating their intentions to implement video consultations.

How might it impact on clinical practice in the foreseeable future

1) Going forward, there is going to be an increase in virtual clinics with a reduction in F2F consultations

2) To achieve patient satisfaction whilst reducing pressure on NHS resources and services, consultation modality will need to depend on several factors: new or follow up patients, routine or urgent follow up, the need for physical examinations, and patient's specific needs such as hearing impairment, language and/or learning disabilities. 


\section{Introduction:}

Inflammatory bowel disease (IBD) is chronic inflammation of the GI tract caused by an immune dysregulation ${ }^{1}$. Patients with IBD are traditionally monitored three to six-monthly in face to face (F2F) outpatient clinics regardless of their disease activity ${ }^{2}$. F2F consultations provide a way of maintaining specialist-patient contact but it is also thought to be an expensive and inefficient way to manage long-term stable conditions ${ }^{3}$. IBD patients in remission with low risk of complications may not necessarily need to attend hospital when they are well but still require long-term specialist monitoring for complications and surveillance needs ${ }^{3}$. Unfortunately, these patients use a significant proportion of outpatient capacity $^{3}$, displacing high-risk or unwell patients who require access to hospital-based specialist services ${ }^{5}$.

There has been increasing pressures within all healthcare systems to reduce the increasing pressures on outpatient resources whilst maintaining a high quality of care towards patients, which is safe and cost-effective ${ }^{4}$. Strategies to reduce this burden include the introduction of virtual clinics, or consultations with patients that take place over the telephone or video. However, virtual clinics require a degree of patient empowerment, and potentially costly IT, nursing services, and administrative support ${ }^{4}$. Reasonable concerns have also been expressed that they may be clinically risky, and/or less acceptable to patients and staff ${ }^{5}$.

Service redesign within the NHS generally occurs over many stages, which includes identifying a need for change, implementing a pilot study and obtaining and responding to feedback from the appropriate stakeholders ${ }^{6}$. Change is a slow and steady process with many variables that need to be factored in before it can be employed permanently. However, since the onset of the COVID-19 pandemic with the United Kingdom being placed under 'lockdown' on March $23^{\text {rd }}, 2020$, there was a rapid restructuring and reorganising of hospital resources throughout the UK National Health Services (NHS) ${ }^{7}$. To minimise transmission of this highly contagious 
virus, there was a rapid implementation of telephone and video consultations in exchange for F2F consultations throughout the NHS.

The British Society of Gastroenterology (BSG) and Crohn's and Colitis UK (CCUK) Foundation published guidance for managing patients with IBD to take into account their perceived higher risk of contracting and developing complications of COVID-198,9. These patients were advised to stay at home, avoid public gatherings and refrain from coming to hospital if possible ${ }^{10}$. Together with the NHS wide shift this resulted in the rapid switch of all IBD outpatient from $\mathrm{F} 2 \mathrm{~F}$ consultations to virtual clinics ${ }^{11}$. Although these changes were initially invoked from this unprecedented pandemic, it appears these changes are here to stay ${ }^{12}$ : virtual clinics are to become the new norm and HCPs and patients alike must all adapt to these changes. We have thus taken this opportunity to assess and evaluate the impact of a virtual clinic service with a detailed analysis on patient and health care professionals (HCPs) satisfaction outcomes, outlining potential enablers and barriers of this adapted service.

Methods and Materials:

\section{Study Population}

This was a multi-centre study from May 1 to July 31 2020. Surveys were distributed to four hospitals in the West Midlands with a mixture of district general and tertiary care hospitals. During this time, a total of 1000 patients awaiting a F2F appointment were automatically switched to telephone consultations. All patients who received a telephone consultation during the months outlined received a survey request to complete. This was an entirely anonymous survey with no identifiable material or information collected. Specific or individual consent was not obtained as the patients were participating without providing any identifiable material. 
A second survey was distributed to IBD HCPs around the UK. This included consultants, nurses, registrars and clinical fellows who were involved in the care of IBD patients during the pandemic.

\section{Study Design}

The patient survey was created online via Google Forms and the online link was sent via text or email. If a mobile number was unavailable, paper surveys were posted. Two weeks after the initial survey was texted to patients, a reminder text to complete the survey was sent. The survey included several sections to collect demographic data and responses on: i) patients' most recent telephone consultation; ii) future consultations; iii) barriers to telephone consultations.

The HCP survey was created online via Google Forms and the online link was sent through deanery and NHS emails and online social platforms such as Twitter and WhatsApp groups. This survey was distinct from the patient survey and included sections on: i) Pre-COVID19 consultations; ii) consultation changes made during COVID19, iii) adaptations for future consultations. IBD HCPs who responded to this survey may not have been involved in the care of the patients who responded to the patient survey.

Both surveys are included in the supplementary files. The surveys were pilot tested on a group of gastroenterologists $(n=6)$ to check the consistency and appropriateness of the questions designed by the investigators. The study was approved by local approval processes and registered as a quality improvement project (QiP).

\section{Statistical Analysis}

Data was analysed using Microsoft Excel 2019 (Microsoft, Redmond, WA, USA) and Stata v16. Categorical and ordinal variables were summarised using counts and percentages and continuous data are presented as medians and interquartile range. Categorical data were analysed using chi-squared tests and one sample paired t-test was used to compare preferences between different modalities of consultation. Variables identified as significant identified by the univariate analysis were used to build a multivariate model to assess the 
effect of each potential confounding factors and to determine independent and significant factors. Linear and logistic regression analysis were used as appropriate to determine independent and significant factors. Dependent variables were modes of consultation and independent variables included demographics, disease characteristics, medications and BSG risk group. All tests were 2 -tailed, a $\mathrm{P}$ value of less than 0.05 was considered statistically significant.

$\underline{\text { Results }}$

\section{Patient Survey Results}

Of the 1000 surveys sent, a total of 210 patients completed the survey (160 online and 50 paper surveys), yielding an overall response rate of $21 \%$. The median age of patients was 55 years (IQR 23), with the majority of replies from females (53.8\%) and of White ethnicity (84.3\%) (See supplementary results section). 107 (51\%) patients had their telephone consultation with an IBD consultant, 49 (23.3\%) with a nurse specialist, 24 (11.5\%) with a registrar and 30 (14.3\%) were unsure who led the consultation.

\section{Healthcare Professional Survey Results}

A total of 114 responses were provided from HCPs across the UK, which included 51 (44.7\%) consultants, 53 (46.5\%) IBD specialist nurses, 6 (5.3\%) registrars and 4 (3.5\%) fellows. PreCOVID, 51\% were already undertaking telephone consultations, of which $39 \%$ (24) were doctors and 64\% (34) were IBD nurse specialists. 2\% (2) were doing video consultations, undertaken by consultants only. During COVID, there was a clear shift towards telephonebased consultations with $88 \%$ of HCPs conducting telephone consultations. For new patient consultations, there was a significantly greater use of telephone $(53 \%)$, video $(1 \%)$ or a mixture of consultations (39\%) compared to F2F alone (7\%); $p<0.001$. There was also a significantly lower use of video consultations over telephone consultation or mixture of consultations $(p<0.001)$. For follow up patients, there was a significantly greater use of 
telephone $(69 \%)$ over F2F $(2 \%)$ or mixture of consultations $(27 \%) ; p<0.001$. There was a significantly lower use of video consultations (2\%) over telephone consultation or mixture of consultations ( $p<0.001$ ). If $F 2 F$ consultations were arranged, $61 \%$ of HCPs had a maximum limit on how many patients could be seen in a morning or afternoon clinic with the average number being 4 patients. Local Trust policies prohibited 15\% of HCPs from seeing any patients F2F.

\section{Telephone consultation vs F2F}

$66.7 \%$ thought they had been thoroughly assessed over the phone with $82.9 \%$ agreeing that their concerns were adequately addressed over the phone. Despite this, only $42.4 \%$ rated their telephone consultation as 'Excellent'. 70.5\% (148) patients said their telephone consultation was 'Just as Good' as a F2F consultation, while $11.9 \%$ (25) said it was overall better and $17.6 \%$ (37) said it was overall worse (Figure 1a). There was a mixed response from HCPs of whether telephone consultations were preferred over F2F, with $52.6 \%$ stating telephone consultations were worse than F2F and $43.9 \%$ stating they were 'just as good' (Fig 1b). Clinicians (consultants, registrars and fellows) thought telephone consultations were worse than F2F (67.2\%), as opposed to nurses (35.8\%). However, neither group thought telephone consultations were particularly better than F2F ( $4.9 \%$ clinicians vs $1.9 \%$ nurses).

\section{Consultation Preferences and Future Intentions}

Overall, patients preferred F2F consultations over telephone or video consultation ( $\mathrm{p} \leq 0.01)$. F2F consultation was consistently preferred over different combinations of telephone, F2F or video consultations $(p<0.001)$. Telephone consultation alone or combination of telephone and F2F was however preferred over video consultations alone or a combination of video and F2F consultations $(p<0.001) .50 \%$ indicated they did not want the option of a video consultation. See Figure 2a for patients' preference for consultation type.

Fewer HCPs want to increase their telephone consultations activity post COVID (Fig 2b); $\mathrm{p}<0.001$. However, significantly fewer want to use F2F alone $(5.3 \%)$ for follow up of IBD patients, preferring the use of telephone $(20.2 \%)$ or combinations of telephone/F2F (22.8\%) or telephone/video consultations (4.4\%); $p<0.05 .34 .2 \%$ of HCPs intend to use a combination 
of all three consultation modalities, with $63 \%$ indicating they intend to use video consultations in the future.

Caucasian ethnicity was found to positively correlated $(R=2.2, p=0.028)$ and patients within the high-risk group negatively correlated $(R=1.01 ; p=0.04)$ with a preference for telephone consultation. Older age $(R=0.03, p=0.02)$ was positively correlated with a preference for combined video and F2F consultation. Older age $(R=0.03 p=0.01)$ and patients with a university degree or higher qualification negatively correlated $(R=-0.81, p=0.04)$ with $F 2 F$ consultations. Having no formal qualification positively correlated $(R=0.98, p=0.02)$ with $F 2 F$ consultations.

Consultation preference based on health status

Table 1 shows patients preference depending on their health status. Unless their IBD was stable, they preferred a F2F consultation, particularly if they were having a flare or needed a change in medication. Video consultations were not preferred in any scenario.

Table 1: Patients preference of consultation based on their health status

\begin{tabular}{|l|l|l|l|l|}
\hline & Telephone & F2F & Video & $\begin{array}{l}\text { Remote review by } \\
\text { Clinical portal/email }\end{array}$ \\
\hline $\begin{array}{l}\text { If patients were ill with a flare of } \\
\text { their IBD }\end{array}$ & $28.6 \%$ & $61.9 \%$ & $9.5 \%$ & N/A \\
\hline $\begin{array}{l}\text { If patients were stable and } \\
\text { needed routine review }\end{array}$ & $68.1 \%$ & $20 \%$ & $11.4 \%$ & $0.5 \%$ \\
\hline $\begin{array}{l}\text { If a change in medication was } \\
\text { required }\end{array}$ & $42.4 \%$ & $49 \%$ & $8.6 \%$ & N/A \\
\hline
\end{tabular}

IBD: Inflammatory bowel disease; F2F: face to face consultations; N/A: Not applicable

\section{Video consultations}

$46.8 \%$ of those over the age of 50 years were agreeable to having future video consultations. This is compared to $53.3 \%$ of those aged less than 50 years $(p=0.86) .71 .2 \%$ of individuals with a university degree or higher were agreeable for a video consultation as opposed to $42.7 \%$ 
without a higher qualification ( $p=0.9) .46 .6 \%$ were agreeable for a video consultation in the White population whereas $67.7 \%$ agreed in the Black, Asian and minority ethnic (BAME) group $(p=0.97)$. Regardless of age, education or ethnicity status, the top ranked consultation preference remained F2F, followed by telephone and then video.

\section{Barriers and Enablers}

Although $71.8 \%$ of patients agreed that learning to use telephone or video services would be easy for them and $76 \%$ were highly willing to use the telephone services in the future, $22.4 \%$ were concerned that telephone services were not adequately secure and $45 \%$ were not comfortable discussing their medical problems over the phone, with no difference between the white population (43.75\%) and the BAME group (41.9\%). The reasons behind this listed by patients were the difficulty in building a rapport with the doctor, missing body language cues and the difficulty in articulating their problems over the phone. There was also mention of specific barriers, such as hearing and learning disabilities and poor internet/phone signal in their homes/workplace. A further $47 \%$ stated they did not think they could have the same relationship with their doctor over the telephone as opposed to $\mathrm{F} 2 \mathrm{~F} .11 \%$ admitted that telephone services would be difficult for them as English was not their primary language.

Figure 3 discusses HCPs overall experience with telephone consultations and difficulties encountered with telephone consultations. Although the majority of HCPs (86\%) were happy to continue to use telephone consultations in the future and thought they were a satisfactory way of conducting a clinical review (79.8\%), 71.9\% felt the breadth of discussion was greater with F2F than telephone consultations. Moreover, $43 \%$ did not think clinics ran quicker with telephone versus F2F. The most common difficulty encountered was poor reception with the line cutting off or going directly to voicemail.

\section{Advantages and Disadvantages}

The most common advantages to telephone clinics that patients described was the reduced travel and wait times, whilst also saving on car park fees. Others commented on not needing to take time off work or arrange childcare. The most common disadvantage listed by patients and HCPs was the inability to carry out a physical examination. Patients also complained that 
they still needed to come into hospital to have blood tests done while HCPs had privacy concerns of being in a noisy office or being interrupted during clinic.

\section{Discussion:}

The BSG have recently released recommendations for restarting outpatient appointments for gastroenterology and hepatology patients post-COVID ${ }^{3}$. They propose to continue to use telephone and video consultations in the future with a decrease in F2F consultations ${ }^{12}$. This is in keeping with the responses from the HCPs from our survey but is in contrast to our patient cohort preference. Virtual clinics can offer many benefits including potential savings to the NHS such as staffing and administrative costs ${ }^{5}$. It can also provide patients with easier and more frequent access to their health care providers ${ }^{13}$. This could have positive effects on compliance and communication and overall improvement in the doctor-patient relationship ${ }^{14}$.

Despite this, many of our patients still preferred the more personal nature of a F2F consultation. There could be multiple reasons behind this sentiment. Rutherford et al found that $35 \%$ of patients would be uncomfortable sharing personal information about their health in a virtual setting ${ }^{15}$, which is in keeping with our results. Greenhalgh et al demonstrated that virtual consultations work better when the patient and clinician know each other ${ }^{16}$. However, this may not be possible as IBD patients are not always seen by the same HCP but rather are managed by a team of specialist consultants, IBD specialist nurses and rotating trainee specialist registrars. This is an important factor considering $14 \%$ of our patients were unaware of who led their phone consultation and highlights the necessity of good communication when undertaking telephone consultations. These barriers may potentially be managed better by the implementation of video consultations, which can provide additional visual information, diagnostic cues and therapeutic presence ${ }^{17}$. Randomised trials have demonstrated video consultations to be associated with higher satisfaction among patients and staff with no difference in disease progression, no substantial difference in service use and lower transaction costs compared with traditional clinic-based care. However, the 
majority of these studies were underpowered with a selected patient population of chronic, stable conditions. These results would not be generalisable to our IBD cohort who can present with acute and potentially serious illnesses. ${ }^{17,18}$ Thus, video consultations, if implemented, should supplement and not replace telephone or F2F consultations.

This paper is the first to highlight the views and opinions of patients and HCPs on the rapid implementation of non F2F consultations for IBD patients during COVID-19. Our study has highlighted two important points, one from the patient's perspective and the other from the clinician's perspective. Firstly, although patients would prefer F2F consultations, they would continue to use telephone consultations if offered but video consultations were the least preferred modality. This is in stark contrast to our HCPs response $(63 \%)$ who intend to increase the amount of video consultations in the future. However, apart from $2 \%$ of our HCP population, video consultations have not yet been trialled by either patients or HCPs. Thus, until this mode of consultation is enacted into clinical practice with further comprehensive analysis, we cannot definitely conclude on its future use.

The second point is that clinicians are moving away from a single modality of consultation and rather intend to combine a mixture of $\mathrm{F} 2 \mathrm{~F}$, video and telephone consultations for the future. Whereas previously, all patients were being seen $\mathrm{F} 2 \mathrm{~F}$ regardless of routine, new or urgent follow-up, consultation modality should now depend on several factors. This includes whether it is a new or follow up patient, the need for a physical examination, the patient's health status and patient's specific needs such as hearing impairment or language difficulties. As each patient is unique with their own set of complexities and needs, one must triage each patient individually to create a balance between patients' preferences and clinicians' intentions with a cost and resource effective NHS service

There are limitations to this study. Firstly, our survey was predominantly completed by the White population (84.3\%), which may bias the results. Only $11 \%$ commented that they would struggle with telephone and video consultation as English is not their primary language. Again, this may be an under-representation as the vast majority of our survey was completed via mobile text link to an online website so those who responded, would not only have access to internet services, but also have a basic understanding of English to complete the survey. 
Therefore, these patients would be more willing to engage with telephone/video consultations. Finally, both our patients and HCPs only had experience with telephone consultations to compare with $\mathrm{F} 2 \mathrm{~F}$ and their views on video consultations are likely to be perception-based and are inconclusive without further studies.

\section{Conclusion:}

Virtual clinics are not a novel approach to delivering outpatient appointments in healthcare. However, IBD is a complex subspecialty with patients often requiring a multidisciplinary management approach and this needs to be taken into consideration when adopting nontraditional consultations. Although HCPs plan to continue with virtual clinics in the future, this should be balanced proportionately with F2F clinics according to patients' clinical needs and preferences.

\section{Figure Legends}

Table 1: Patients preference of consultation based on their health status. IBD: inflammatory bowel disease; F2F: face to face consultations; N/A: Not applicable

Figure 1: a) Patients and b) Healthcare professionals' response to how they compared their telephone consultation to a F2F consultation. F2F: face to face consultation

Figure 2: a) Patients ranked their top 3 preferences for consultations in the future. b) Healthcare professionals' intentions for consultations in the future.

Figure 3: a) Overall experiences of Healthcare professionals with telephone consultations. b) The difficulties encountered by Healthcare professionals with telephone consultations 


\section{Author contributions:}

AK- Designed the survey, distributed the survey at her trust, collected data and analysed results. She researched the literature and wrote the manuscript. She was involved with manuscript revisions

MNQ- Contributed to the design of the survey and distributed the surveys at his trust. He statistically analysed the results and was involved with critical manuscript revisions SDS, NT \& HS- Contributed to the design of the survey and distributed the surveys at their trust.

MJB- Developed the concept and designed the survey. He was involved with critical manuscript revisions.

RC- Developed the concept and designed the survey. She distributed the surveys at her trust. She was involved with critical manuscript revisions

\section{Competing interest:}

SDS- Speaker fees/ Consultancy- Abbvie, Pfizer, Takeda, Dr Falk, Predictimmune, Janssen SDS- Conference travel- Pfizer, Tillots, Predictimmune, Abbvie, Dr Falk, Janssen NT- has received research support from Astra Zeneca, Dr Falk, MSD and Pfizer. MJB has received grants and travel expenses from Vifor International and Tillots Pharma, outside of the submitted work.

All other authors report no competing interests.

\section{License for Publication}

The Corresponding Author has the right to grant on behalf of all authors and does grant on behalf of all authors, an exclusive licence (or non-exclusive for government employees) on a worldwide basis to the BMJ Publishing Group Ltd to permit this article (if accepted) to be published in BJO and any other BMJPGL products and sublicences such use and exploit all subsidiary rights, as set out in our licence (http://group.bmj.com/products/journals/instructions-for-authors/licence-forms). 
References:

1. Sandberg K, Yarger E, Saeed S. Updated in diagnosis and management of inflammatory bowel disease. Curr Probl Pediatr Adolesc Health Care. 2020; 50: 100785

2. Robinson A, Thompson DG, Wilkin D, et al. Northwest Gastrointestinal Research Group. Guided self-management and patient-directed follow-up of ulcerative colitis: a randomised trial. Lancet2001;358:976-81.

3. Hunter J, Claridge A, James S et al. Improving outpatient services: the Southampton IBD virtual clinic. Frontline gastroenterology. 2012; 3: 76-80.

4. Fofaria, RF, Barber S, Adeleke $\mathrm{Y}$ et al. Stratification of inflammatory bowel disease outpatients by disease activity and risk of complications to guide out-of-hospital monitoring: a patient-centred quality improvement project. BMJ Open Quality. 2019; 8: e000546

5. Greenhalgh T, Vijayaraghavan S, Wherton J et al. Virtual online consultations: advantages and limitations (VOCAL) study. BMJ Open. 2016; 6: e009388.

6. Quality, service improvement and redesign (QSIR) tools by type of task. NHS improvement ACT Academy. Published September 8, 2017. Accessed October 6, 2020. $<$ https://improvement.nhs.uk/resources/quality-service-improvement-and-redesignqsir-tools-type-task/>

7. Gilbert AW, Billany JCT, Adam R et al. Rapid implementation of virtual clinics due to COVID-19: report and early evaluation of a quality improvement initiative. BMJ Open Quality. 2020; 9: e000985.

8. Edwards C \& Lees C.BSG COVID-19 guidance on IBD patient risk groups. British Society of Gastroenterology. https://www.bsg.org.uk/covid-19-advice/bsg-advice-on-ibdpatient-risk-groups/

9. Coronavirus (COVID-19): What your risk means for you. <https://crohnsandcolitis.org.uk/news/advice-for-people-with-crohns-and-colitisself-isolation-social-distancing > Accessed October 4, 2020

10. Monteleone G \& Ardizzone S. Are patients with inflammatory bowel disease at increased risk for covid-19 infection? JCC. 2020; 14(9): 1334-6 
11. Kennedy NA, Jones GR, Lamb CA et al. British society of gastroenterology guidance for management of inflammatory bowel disease during the COVID-19 pandemic. Gut. 2020; 69(6).

12. Rebooting Gastroenterology and Hepatology Outpatients in the wake of COVID-19: Renewal, redesign and establishing the "new norm". British Society of Gastroenterology Guidance. 2020. < https://www.bsg.org.uk/wpcontent/uploads/2020/07/Outpatient-services-restart-and-redesign-Ver-Final-2.pdf> Accessed October 1, 2020

13. Powell RE, Henstenburg JM, Cooper G et al. Patient perceptions of telehealth primary care video visits. Annals of family medicine. 2017; 15(3): 225-229.

14. Ekbom A. The epidemiology of IBD: A lot of data but little knowledge. How shall we proceed? Inflammatory Bowel Diseases. 2004; 10: S32-34

15. Rutherford E, Noray R, O hEarrain C et al. Potential benefits and drawbacks of virtual clinics in general surgery: pilot cross-sectional questionnaire study. JMIR Periper Med. 2020; 3(1): e12491

16. Greenhalgh T, Koh GCH, Car J. Covid-19: a remote assessment in primary care. BMJ. 2020;368:m1182.

17. Greenhalgh T, Wherton J, Shaw S, et al. Video consultations for covid-19. BMJ 2020;368: m998.

18. Ignatowicz A, Atherton $\mathrm{H}$, Bernstein $\mathrm{CJ}$, et al. Internet videoconferencing for patientclinician consultations in long-term conditions: A review of reviews and applications in line with guidelines and recommendations. Digit Health 2019; 5: 2055207619845831. doi:10.1177/2055207619845831 